\title{
INFLATIONARY UNIVERSE, PRIMORDLAL SOUND WAVES AND GALAXY FORMATION
}

\author{
V.N. Lukash and I.D. Novikov \\ Space Research Institute, Moscow
}

\section{Observational data and requirements to theory}

At present cosmology experiences a qualitatively new state of its development. Up to now cosmological theory successfully extrapolated to the past the Universe we observe now. This extrapolation was reliable up to the temperatures $T \sim 10^{10} \mathrm{~K}$. The endeavors to advance nearer to the singularity imply that even cardinally new hypotheses in the elementary particle physics (e.g. Hagerdorn's hypothesis) brought about the relatively insignificant variations in cosmology-the Universe expansion rate obeyed the power law as before, the main features of the cosmological model (homogeneity, singularity, the existence of horizone, etc.) remained unchanged.

The great success of the first stage of cosmology development was a formulation of the initial conditions which should have been prescribed from the very beginning of the cosmological expansion and resulted in the present state of the Universe. These conditions of the early Universe and their relation to the observable world are given below.

1. Spatial homogeneity and isotropy of the cosmological expansion. This property follows from reliably determined large-scale isotropy and homogeneity of the observed world (data on relic radiation, distribution of galaxy clusters, abundance of $\mathrm{He}^{4}$ and so on) which indicate that the Universe expanded in the past in accordance with the Friedmann model. Non-Friedmannian beginning of the expansion attended by isotropization would result in the observed effects which have not been detected so far (isotropization problem, see $[1,2]$ etc.). The fact that the physical conditions are the same in the causally nonrelated space regions, flled up with radiation and matter, cannot be explained in the context of classical cosmology (horizon problem).

2. Primordial cosmological perturbations. The developed structure of the observed Universe (galaxies and their clusters) on scales of $\sim 0.01$ horizon and less along with a high degree of homogeneity and isotropy on larger scales (see 1) indicate that the observed structure of the Universe appeared as a result of the development of small perturbations-deviations from isotropy and homogeneity-which should exist in the beginning of the expansion. At the radiation-dominated stage, when pressure is one-third of the energy density $\left(p=\frac{f}{s}\right)$, the small primordial perturbations are the cosmological sound waves of constant amplitude. For these perturbations to increase to unity in the 
epoch after hydrogen recombination their amplitude at the same $p=\frac{\epsilon}{3}$ should be of the order of $10^{-4}$ on scale of clusters. This is much more than the statistical or quantum density fluctuations. The absence of the large amount of the primordial black holes and of large-scale fluctuations of the relic radiation suggests that the primordial perturbation spectrum is close to the flat one [3].

3. Baryon asy mmetry. The observed world consists of matter, the noticeable presence of anti-matter in the Universe has not been detected. Qualitatively a number of baryons (at the hot stage - their excess over antibaryons) is convenient to characterize relative to relic photons, the number of which is strictly determined in the hot Universe. This ratio- one baryon per $\sim 10^{8}$ photons-is conserved in the process of expansion, insignificantly varying at the epoch of annihilation of electron-positron and other pairs at the hot stage of expansion. Such a high value of specific entropy evidences the hot past of the Universe when the matter was ultrarelativistic with the equation of state $p=\frac{\epsilon}{3}$.

4. Mean density of the matter and spatial flatness of the Universe. The density of the visible matter in the Universe is less than the critical one by the factor of $\sim 30$. Other data show the possible presence in space of invisible matter. The fact that the mean density of the matter is close to the critical one in 15 billion years after the beginning of expansion evidences that the three-dimensional space of the Universe on the horizon scale is practically flat.

5. Absence of $\Lambda$-term. Physical vacuum of all the fields has no gravity.

The modern second stage of the cosmological theory development is aimed to explain these five puzzles of the early Universe. This became possible due to the advances of the elementary particle physics. Spontaneously broken gauge theories (see reviews $[4,5,6]$ and others) realized in a simple mathematical form the idea of unification of physical interactions at very high energies and predicted the presence of phase transitions with breaking of the ground state of quantum fields in the process of cosmological expansion.

Not long before a typical phase transition the Universe expansion is determined by the density of a symmetric "false" vacuum whose energy then converts partly to usual matter. At this stage the Universe expands exponentially fast and for several tens of cosmological times the Planck scale can infiate up to very large sizes, e.g., to thc present visible horizon and even more. During such an expansion all random (chaotic) unhomogeneities and curvatures which could take place at the beginning are ironed, as at this stage the effect of the vacuum is equivalent to the presence of a large $\Lambda$-term, homogeneous in space and as if preserving the future homogeneity, isotropy and flatness of the Universe. At this stage the world approaches the part of de Sitter's world, therefore the inflationary stage is often called de Sitter's.

So, the existence of de Sitter's expansion stages in the early Universe with the successive decrease of the value of $\Lambda$ solves in principle problems 1,4 , and 5 [7]. On the other hand grand unified theories, predicting baryon-and CP-nonconservation intereactions with energies $10^{14}-10^{17} \mathrm{GeV}$, explain successfully question 3 on the origin of baryon asymmetry by the decay of heavy leptons and Higgs' bosons (see, e.g., reviews $[8,9]$ ). To make this baryon number generation possible, de Sitter's expansion stage should have occurred before the moment the world had a temperature of the grand unifcation energy for the last time. The existence of such rather stretched exponential expansion stage could have also explained the absence of superheavy monopoles which 
were formed during the grand unification symmetry breaking since these monopoles would get beyond the visible horizon nowadays [10].

Before discussing the main point of our paper-generation of the primordial cosmological perturbations-it should be mentioned that de Sitter's stage near the cosmological singularity could appear not only due to Higg's breaking which does not effect the gravitational field (the metric is classical and its symmetry does not change) but also, in principle, due to quantum gravitational effects [11,12].

This problem is closely connected with the problem of the cosmological singularity and, to our point of view; only a future theory of quantum gravity can solve it.

\section{Generation of primordial perturbations}

During phase transition at the exponential expansion stage unhomogeneities form spontaneously, it would be desirable to identify them with those primordial (see 2). The resulting spectrum is flat-this property of de Sitter's stage is inherent in both gravitational waves [13] and potential perturbations [14,18]. The question about potential fluctuations amplitude is more problematic since the latter depends on the phase transition kinetics.

It was assumed in the first versions of the theory for the simplest model of Higg's scalar field that the first order phase transition to the stage of a new phase $(\phi \neq 0)$ results at once in the formation of "true" vacuum bubbles (see [4-6]). In this case practically all released energy goes to the walls of nucleated bubbles which, expanding and colliding, form large unhomogeneities contradicting the observations.

In a new version of the theory [10] that differs by a special choice of the effective potential determining the ty pe of the $\phi$-field nonlinearity, the phase transition kinetics is such that a formed bubble continues expanding exponentially and can expand up to the size larger than the Universe observed. In this version 1, and 4 initial condition problems and also the absence of the noticeable number of monopoles and primordial black holes are naturally explained. The termination of de Sitter's stage and the transfer to the radiation-dominated expansion stage are ensured by a decay of $\Lambda$-term inside the bubble with the formation of the "true" vacuum and thermal particles. A rigorous account of this transition stage depending on the type of the effective potential, makes it possible to calculate the value of potential perturbations generated in the bubble. In the new inflationary Universe scenario for the simplest phase transition model the amplitude of potential perturbations also turns out to be too high to be consistent. with observations [15-18].

The amplitude of potential fluctuations created during the phase transition on scales less than the cosmological horizon (mind, that the bubble infiates with the velocity of light) is directly connected with the choice of the efiective potential of Higgs' field considered. In the real Universe the symmetry of physical fields was apparently more complex, during the transition fields could interact intensively, the effective potential could have quite another form than that investigated up to now. Evidenty, there may exist theories with acceptable effective potentials (see, e.g., [19]). Thus, one of the basic problems is to build a theory which does not result in fairly large density fluctuations and baryon charge of the Universe.

In this paper we would like to pay attention to perturbations the genera- 
tion of which does not depend on details of phase transitions, but is rather provided by the existence of the intermediate de Sitter stage. These perturbations are spontaneously generated from the point-zero fluctuations of metric through the parametric amplification effect on scales more or of the order of the cosmological horizon [14] (the latter grows as fast as a scale factor at the de Sitter stage). To determine these perturbations correctly we need some assumptions about the matter since matter and metric perturbations of potential type are strictly coupled [20]. Independently of the choice of a field theory, the matter in such large scales can be described as a perfect fluid with the effective equation of state $p=p(\epsilon)$.

The perturbations of this gravitating fluid are relativistic sound waves described by the gauge invariant 4 -scalar $q(t, x)[14,21]$ :

$$
\square_{\beta} \boldsymbol{q}=U \boldsymbol{q},
$$

where $d s^{2}=d t^{2}-a^{2}\left(d x^{2}+d y^{2}+d z^{2}\right)$ is the metric of background Friedmann model, $a=a(t)$ is the scale factor, $\square_{\beta}=\frac{\partial^{2}}{\partial \eta^{2}}-\beta^{2}\left(\frac{\partial^{2}}{\partial x^{2}}+\frac{\partial^{2}}{\partial y^{2}}+\frac{\partial^{2}}{\partial z^{2}}\right.$ is the acoustic d'Alambertian operator, $\eta=\int \frac{d t}{a}$ is the conformal time, $\beta=\left(\frac{d p}{d \epsilon}\right)^{\frac{1}{2}}$ is the sound velocity, $U=(\xi a)^{-1} \frac{d^{2}}{d \eta^{2}}(\xi a)$ is the sound effective potential reflecting nonstationarity of the Universe, $\xi=\frac{(1+p / \epsilon)^{\frac{1}{2}}}{2 \beta}, q=\xi \leftrightarrow q$. In the short-wave asymptotic $q$ has a meaning of hydrodynamic velocity potential. For large scales being of interest for us $q$ is mainly metric perturbations.

At the hot evolutionary stage when the Universe expansion is determined by relativistic particles, $q$-field proves conformally coupled: $U=0$. At this stage, using the procedure of secondary quantization, the vacuum of $q$-field can be determined, which on large scales corresponds to quantum metric potential perturbations.

The possibility of spontaneous birth of phonons, quanta of the $q$-field, comes due to the fact, that sound effective potential differs from zero at de Sitter stage, $U \neq 0$. This effect works as a typical parametric amplification. The resulting perturbation spectrum somewhat differs from the flat one [22,23]: it damps in a direction of large scales inversely with perturbation size and has a characteristic maximum with the exponential cut in a direction of short-waves, the perturbation amplitude in the maximum is of the order of the temperature squared (in Planck units), at which the Universe was at the beginning of the first de Sitter stage.

For these perturbations could be primordial to form galaxies, the spectrum maximum should be within the mass range from $10^{3} M_{\odot}$ to $10^{15} M_{\odot}$. This requires the total duration of all de Sitter stages from 30 to 60 cosmological times. To ensure the required perturbation amplitude in the scales of clusters the first phase transition should occur not later than in $\sim 10^{4}$ Planck times, that corresponds to the energy of $\sim 10^{17}$ $\mathrm{GeV}$. This figure does not yet contradict to the estimate of the density of gravitational waves, produced due to this transition, which will affect the large-scale isotropy of relict radiation $[13,24,25]$. The induced quadrupole relict anisotropy will be of the same order of magnitude as the density perturbation amplitude in the spectrum's maximum.

1. A.G. Doroshkevich, V.N. Lukash, I.D. Novikov, Zh. Eksp. Teor. Fiz. 64, 1457 (1973).

2. C.B. Collins, S.Q. Hawking, Astroph. J. 180, 317 (1973). 
3. Ya.B. Zeldovich, Mon. Not. R. Astron. Soc. 160, 1L (1972).

4. S. Dimopoulos, Proc. of IAU Symposium 104 "Early Evolution of the Universe and Its Present Structure," Crete, Greece, Aug 30-Sep 2 (1982).

5. K. Sato, Proc. of IAU Symposium 104 "Early Evolution of the Universe and Its Present Structure," Crete, Greece, Aug 30-Sep 2 (1982).

6. S. Bludman, Proc. of IAU Symposium 104 "Early Evolution of the Universe and Its Present Structure," Crete, Greece, Aug 30-Sep 21982 , (1983) D. 447.

7. A.H. Guth, Phys. Rev. D2s, 347 (1981).

8. A.D. Dolgov, Ya.B. Zeldovich, Rev, Mod. Phys. 53, 3 (1981).

9. M. Turner, Proc. of LAU Symposium 104 "Early Evolution of the Universe and Its Present Structure," Crete, Greece, Aug 30-Sep 2 (1982).

10. A.D. Linde, Phys. Lett. 108B, 389 (1982).

11. A.A. Starobinski, Phys. Lett. 91B, 99 (1980).

12. L.P. Grishchuk, Ya.B. Zeldovich, Proc. of 2nd Seminar "Quantum Gravity," Moscow, Oct 13-15 (1981).

13. A.A. Starobinski, Pis the Zh. Eksp. Teor. Fiz. 80, 719 (1979).

14. V.N. Lukash, Zh. Eksp. Teor. Fiz. 79, 1601 (1980).

15. J.M. Bardeen, Proc. of Nuffield Workshop on the Very Early Universe $(<1 \mathrm{sec})$, Cambridge, June 21-July 9 (1982).

16. A.H. Guth, Proc. of Nuffield Workshop on the Very Early Universe $(<1 \mathrm{sec})$, Cambridge, June 21-July 9 (1982).

17. S.W. Hawking,Proc. of Nuffield Workshop on the Very Early Universe $(<1 \mathrm{sec})$, Cambridge, June 21-July 9 (1982).

18. A.A. Starobinski, Proc. of Nuffield Workshop on the Very Early Universe $(<1 \mathrm{sec})$, Cambridge, June 21-July 9 (1982).

19. P.J. Steinhardt, Proc. of Nuffield Workshop on the Very Early Universe $(<1 \mathrm{sec})$, Cambridge, June 21-July 9 (1982).

20. E.M. Lifshitz, Zh. Eksp Teor. Fiz. 16, 587 (1946).

21. V.N. Lukash, Pis tma Zh. Eksp. Teor. Fiz. 81, 631 (1980).

22. D.A. Kompaneets, V.N. Lukash, I.D. Novikov, Astron. Zh. 50, 424 (1982).

23. V.N. Lukash, I.D. Novikov, Proc. of Nuffield Workshop on the Very Early Universe (<1 sec), Cambridge, June 21-July 9 (1982).

24. L.P. Grishchuk, Zh. Eksp. Teor. Fix. 67, 825 (1974).

25. A.V. Veryaskin, V.A. Rubakov, M.V. Sazhin, submitted to Phys. Lett. $B(1982)$.

Discussion

B. Jones: Are there any dissipative processes, occurring perhaps when the $\mathrm{X}$-bosons decouple, which could inhibit or damp the growth of the fluctuations which you created at earlier times?

Novikov: We are interested in the fluctuations, the scale of which is much greater than the horizon at the early universe. These perturbations are not influenced by any matter effects, in particular by dissipative processes.

Bonometto: I would like to understand why the equation for sound wave propagation contains a term violating conformal invariance during the exponential expansion, while this term is not there before and 
after. In other terms, a characteristic scale enters the equation during the inflationary phase. What is this scale?

Novikov: The effective potential of sound waves $U=\alpha " \alpha=$ (time scale to the minus two) is exactly equal to zero, when the universe expands linearly in the conformal time $\alpha \sim n$ (it takes place for the $p=\varepsilon / 3$ equation state), and it appears non-zero when the expansion deviates from the linear law, e.g., when it is exponential. A characteristic scale here is the scale of the horizon at the beginning of the inflationary stage. If the latter is provided by GUTs, then the characteristic scale turns out to be the GUT scale, because the temperature at the beginning of the exponential stage is of the order of the "vacuum" density, $\alpha=$ (scale factor) $(1+$ pressure/density) $1 / 2$ $/ 2$ (sound velocity). 